\title{
Influência da Mica nas Propriedades Mecânicas e Dinâmico-Mecânicas de Composições de Borracha Natural
}

\author{
Viviane A. Escócio, Agnes F. Martins, Leila L.Y. Visconte, Regina C.R. Nunes \\ IMA, UFRJ \\ Djanira M. R. Costa \\ Instituto Nacional de Tecnologia (INT), RJ
}

Resumo: Neste trabalho foi estudada a influência da adição de mica em composições vulcanizáveis de borracha natural quanto às propriedades mecânicas e dinâmico-mecânicas. As diversas composições também foram avaliadas quanto à densidade de ligações cruzadas. Todos os dados foram comparados aos da composição sem carga. Os resultados mostraram que a adição de teores crescentes de mica na borracha natural aumentou o módulo a 300\%, a dureza, a densidade de ligações cruzadas e a deformação permanente à compressão (DPC) das composições, por outro lado diminuiu a resiliência e a resistência à abrasão. O melhor desempenho quanto à resistência ao rasgamento e à tensão na ruptura foi obtido com 30phr de mica. As propriedades dinâmico-mecânicas (DMTA) corroboraram as propriedades mecânicas obtidas.

Palavras-chave: Borracha natural, mica, propriedades físico-mecânicas, propriedades dinâmico-mecânicas.

\section{Influence of Mica on Mechanical and Dynamic Mechanical Properties of Natural Rubber Compositions}

Abstract: In this work the influence of mica on the mechanical and dynamic mechanical properties of vulcanized natural rubber compositions was studied. The crosslinking density of the compositions was also determined. The data were compared to those of the unfilled composition. The results indicate an increase in modulus, hardness, crosslinking density and compression set as well as a decrease in resilience and abrasion resistance for the natural rubber compositions with increasing mica contents. The best performance for tear strength and stress at break was achieved with $30 \mathrm{phr}$ of mica. The dynamic mechanical behavior of the compositions (DMTA) is in agreement with their mechanical properties.

Keywords: Natural rubber, mica, physical mechanical properties, dynamic mechanical properties.

\section{Introdução}

A borracha natural (NR) é um dos polímeros mais consumidos mundialmente devido à combinação de suas únicas e excelentes propriedades, tais como elevada resistência à tração e ao rasgamento e excelentes propriedades dinâmicas $^{[1]}$. A competição entre a NR e os elastômeros sintéticos se concentra nas propriedades oferecidas, e além disso no preço e na sua disponibilidade ${ }^{[2,3]}$.

Ao projetar um compósito elastomérico para aplicação específica também é importante considerar o processamento e o preço do artefato final. As propriedades finais da NR dependem do grau de vulcanização, da formulação escolhida, do tipo e da quantidade de carga. Algumas vezes a cor, transparência ou opacidade são também importantes. É de conhecimento geral que, para melhorar o desempenho mecânico e baratear o produto, a escolha de cargas é de grande relevância, principalmente no caso de elastômeros que têm baixo módulo ${ }^{[4]}$.

$\mathrm{O}$ negro de fumo é a carga mais usada para aumentar o desempenho mecânico de compósitos elastoméricos ${ }^{[1]}$. Ainda que o negro de fumo não tenha baixo custo, seu preço é inferior ao da sílica, que é a carga competitiva na melhoria das propriedades mecânicas de elastômeros ${ }^{[4]}$. Uma das vantagens da sílica é a cor clara, permitindo que o artefato tenha colorações diferenciadas. Outros materiais são empregados como cargas claras em borrachas, como argilas ou carbonatos, porém com desempenho tecnológico inferior ${ }^{[1]}$.

A mica vem sendo muito utilizada como carga em termoplásticos e termorrígidos, porém em elastômeros seu uso é menos estudado. A importância do emprego da mica em polímeros de um modo geral, deve-se ao seu baixo custo e abundância no Brasil, além de suas ótimas propriedades de isolamento elétrico e térmico ${ }^{[5,6]}$.

Mica é o nome de um grupo de minerais filossilicatos, de fórmula geral $\mathrm{KM}\left(\mathrm{AlSi}_{3} \mathrm{O}_{10}\right)(\mathrm{OH})_{2}$, onde $\mathrm{M}$ deve ser $\mathrm{Al}, \mathrm{Fe}, \mathrm{Mg}$ ou combinações destes metais ${ }^{[7-10]}$. $\mathrm{O}$ grupo da mica tem cerca de 30 membros, mas somente três são comercialmente importantes: moscovita, flogopita e biotita $^{[7,9,11]}$.

Em geral as micas apresentam excelente resistência a intempéries, boa resistência química, baixa condutividade térmica, estabilidade em altas temperaturas, características de não inflamabilidade, baixa condutividade elétrica, alta

Autor para correspondência: Regina C. R. Nunes, IMA, UFRJ, Caixa Postal 68525, CEP: 21945-970, Rio de Janeiro, RJ. E-mail: rcnunes@ima.ufr.br 
rigidez dielétrica, baixo fator de dissipação e baixo custo ${ }^{[12]}$. Trabalhos com NR estudaram o desempenho da mica na redução da permeabilidade de ar na borracha ${ }^{[13]}$ e no aumento da retardância à chama dessa borracha ${ }^{[14]}$, sem perda de suas propriedades mecânicas.

Os artefatos de borrachas são freqüentemente usados em aplicações onde ocorrem rápidas deformações cíclicas em certa freqüência, ou em uma faixa de freqüências, como é o caso, por exemplo, das bandas de rodagem de pneus. As propriedades dinâmico-mecânicas são especificamente aplicadas nestes casos, pois envolvem a determinação do comportamento de materiais sujeitos a movimentos oscilatórios, em deformações que podem ser tensão, flexão, cisalhamento ou compressão. Como as borrachas são viscoelásticas, o teste dinâmico-mecânico proporciona um método ideal para a sua caracterização, permitindo a determinação do módulo elástico (E') e módulo viscoso (E") em função da temperatura, freqüência ou tempo ${ }^{[15]}$.

Neste trabalho foram desenvolvidas composições vulcanizáveis de borracha natural, com teores variados de mica entre 0 e $40 \mathrm{phr}$. Todas as composições foram caracterizadas quanto às propriedades físico-mecânicas e dinâmicomecânicas.

\section{Experimental}

As composições de borracha natural foram preparadas em moinho de rolos Berstorff, a $65{ }^{\circ} \mathrm{C}$, com razão de fricção de 1:1,25. A formulação (em phr) usada seguiu a norma ASTM D 3184: NR (100); ZnO (5,0); S (2,25); ácido esteárico (2,0); TBBS (N-t-butil-2-benzotiazol sulfenamida) $(0,7)$; Irganox 1010 (tetra-[3-(3',5'-di-t-butil-4'hidroxifenil)-propionato] de pentaeritritol) $(1,0)$. O teor de mica incorporado variou entre 0 e $40 \mathrm{phr}$, e esta carga foi seca a $120^{\circ} \mathrm{C}$ durante 1 hora, antes da etapa de mistura. As características físico-químicas da mica se encontram na Tabela 1. A borracha natural usada foi do tipo 1 GEB (granulado escuro brasileiro), com viscosidade Mooney 95,4 ML (1+4) a $100{ }^{\circ} \mathrm{C}$, obtida de acordo com a norma ASTM D 1646.

A determinação dos parâmetros reométricos foi feita em reômetro de disco oscilatório da marca Tecnología Industrial, modelo TI-100, de acordo com norma a ASTM D 2084. Os parâmetros de vulcanização avaliados foram: torque mínimo $\left(\mathrm{M}_{\mathrm{L}}\right)$, torque máximo $\left(\mathrm{M}_{\mathrm{H}}\right)$, tempo de pré-vulcanização $\left(\mathrm{t}_{\mathrm{s}} 2\right)$, tempo de vulcanização a $90 \%\left(\mathrm{t}_{90}\right)$ e índice de velocidade de cura (CRI). As condições utilizadas para as análises foram: temperatura, $160^{\circ} \mathrm{C}$; arco de oscilação, $3^{\circ}$; e tempo, 1 hora. As análises de cada composição foram feitas em triplicata, e os resultados foram a média aritmética dos valores parciais.

Os ensaios de tração e rasgamento foram realizados em dinamômetro Instron, modelo 1101, segundo as normas ASTM D 412 e D 624, respectivamente, à temperatura ambiente e velocidade de separação das garras de $500 \mathrm{~mm} /$ min. Para o ensaio de rasgamento foram usados corpos de prova modelo C. Os corpos de prova foram extraídos de placas, vulcanizadas a partir dos valores de $t_{90}$. Para esses ensaios
Tabela 1. Propriedades físico-químicas da mica ${ }^{[4]}$

\begin{tabular}{|c|c|c|}
\hline \multicolumn{3}{|c|}{ Mica tipo Moscovita } \\
\hline Análise química ${ }^{(a)}$ & \multicolumn{2}{|c|}{$\%$} \\
\hline $\mathrm{SiO}_{2}$ & \multicolumn{2}{|c|}{53,00} \\
\hline $\mathrm{Al}_{2} \mathrm{O}_{3}$ & \multicolumn{2}{|c|}{37,27} \\
\hline $\mathrm{K}_{2} \mathrm{O}$ & \multicolumn{2}{|c|}{7,53} \\
\hline $\mathrm{Fe}_{2} \mathrm{O}_{3}$ & \multicolumn{2}{|c|}{0,67} \\
\hline $\mathrm{Na}_{2} \mathrm{O}$ & \multicolumn{2}{|c|}{0,39} \\
\hline $\mathrm{TiO}_{2}$ & \multicolumn{2}{|c|}{0,24} \\
\hline $\mathrm{Pb}_{2} \mathrm{O}_{5}$ & \multicolumn{2}{|c|}{0,07} \\
\hline $\mathrm{MgO}$ & \multicolumn{2}{|c|}{0,07} \\
\hline $\mathrm{CaO}$ & \multicolumn{2}{|c|}{0,03} \\
\hline Perda ao fogo & \multicolumn{2}{|c|}{0,25} \\
\hline Propriedades físicas ${ }^{(b)}$ & $\left(\mathrm{g} / \mathrm{cm}^{3}\right)$ & $(\mu \mathrm{m})$ \\
\hline Peso específico & 2,70 & - \\
\hline Diâmetro médio de partículas & - & 18,00 \\
\hline Densidade aparente & $0,45-0,75$ & - \\
\hline
\end{tabular}

(a) determinado experimentalmente

(b) dados fornecidos pelo fabricante

foram usados 5 corpos de prova de cada composição, sendo os resultados as medianas dos valores parciais.

O ensaio de dureza foi realizado segundo norma ASTM D 2240 em durômetro Shore, tipo A-2. Foram feitas 5 medidas para cada composição e os resultados foram as medianas dos valores parciais. Outras propriedades mecânicas estudadas foram resiliência, resistência à abrasão e deformação permanente à compressão, realizadas de acordo com as normas ASTM D 2632, D 1044 e D 395, respectivamente. Para esses ensaios as análises foram feitas em duplicata e os resultados foram a média aritmética dos valores parciais.

A densidade de ligações cruzadas foi calculada segundo a equação desenvolvida por Flory-Rehner, tendo por base o inchamento no equilíbrio em solventes orgânicos ${ }^{[16]}$. O solvente usado neste trabalho foi o heptano, sendo o equilíbrio atingido após 7 dias, em temperatura ambiente e no escuro. Os corpos de prova utilizados tinham dimensões de $2,0 \times 2,0 \times 0,2 \mathrm{~cm}$ sendo o resultado a média aritmética de duas determinações.

A análise termo-dinâmico-mecânica foi realizada em analisador DMTA da marca Rheometric Scientific, modelo MK III, nas seguintes condições: modo de deformação, flexão; freqüência, $1 \mathrm{~Hz}$; velocidade de aquecimento, $2^{\circ} \mathrm{C} / \mathrm{min}$; e faixa de temperatura, -80 a $20^{\circ} \mathrm{C}$.

Neste trabalho, todos os materiais foram usados como recebidos para não onerar o preço da composição final. 
Tabela 2. Parâmetros de vulcanização das composições NR/mica

\begin{tabular}{cccccc}
\hline $\begin{array}{c}\text { Teor de mica } \\
(\mathbf{p h r})\end{array}$ & $\begin{array}{c}\mathbf{M}_{\mathbf{L}} \\
(\mathbf{d N} . \mathbf{m})\end{array}$ & $\begin{array}{c}\mathbf{M}_{\mathbf{H}} \\
(\mathbf{d N} . \mathbf{m})\end{array}$ & $\begin{array}{c}\mathbf{t}_{\mathbf{s}} \mathbf{2} \\
(\mathbf{m i n})\end{array}$ & $\begin{array}{c}\mathbf{t}_{\mathbf{9 0}} \\
(\mathbf{m i n})\end{array}$ & $\begin{array}{c}\mathbf{C R I} \\
\left(\mathbf{m i n}^{-1}\right)\end{array}$ \\
\hline 0 & 10,2 & 49,8 & 3,0 & 8,4 & 18,52 \\
10 & 8,2 & 51,3 & 2,4 & 8,0 & 17,86 \\
20 & 7,0 & 49,2 & 2,4 & 8,2 & 17,24 \\
25 & 6,0 & 51,4 & 2,3 & 7,0 & 21,28 \\
30 & 5,5 & 53,6 & 2,4 & 6,5 & 24,39 \\
35 & 5,5 & 54,0 & 3,0 & 7,8 & 20,83 \\
40 & 7,8 & 56,3 & 3,0 & 7,7 & 21,28 \\
\hline
\end{tabular}

\section{Resultados e Discussão}

Os parâmetros de vulcanização das composições de borracha natural estudados estão apresentados na Tabela 2. De forma geral pode ser observado que, em relação à composição sem carga, a adição de mica causa uma diminuição do torque mínimo $\left(\mathrm{M}_{\mathrm{L}}\right)$, do tempo de pré-vulcanização $\left(\mathrm{t}_{\mathrm{s}} 2\right)$ e do tempo de vulcanização a $90 \%\left(t_{90}\right)$ das composições carregadas, assim como provoca um aumento do torque máximo $\left(\mathrm{M}_{\mathrm{H}}\right)$. Em relação ao torque mínimo, menores valores deste parâmetro indicam que a mica facilita o processamento da borracha natural. O aumento do torque máximo pela adição de carga é um efeito esperado e está relacionado ao aumento da rigidez da matriz elastomérica após a vulcanização. Esta maior rigidez pode ser devido tanto à presença da carga, quanto à formação de um maior número de ligações cruzadas (químicas e/ou físicas), o que não implica em melhores propriedades mecânicas para esses materiais. Os valores mais baixos de $t_{s} 2$ e $t_{90}$ mostram que a mica favorece a vulcanização da borracha natural, que se inicia antes e é mais rápida, o que pode ser comprovado pelos maiores valores do índice de velocidade de cura (CRI) a partir de $25 \mathrm{phr}$ de mica. Os resultados obtidos também indicam ser em torno de $30 \mathrm{phr}$, o valor limite para a carga em estudo.

A Figura 1 mostra os resultados de tensão na ruptura, alongamento na ruptura e resistência ao rasgamento das composições estudadas. Pode ser observado que a tensão na ruptura e a resistência ao rasgamento têm em comum $30 \mathrm{phr}$ de mica como limite superior de carga. $\mathrm{O}$ alongamento na ruptura diminui com o aumento do teor de mica, como era de se esperar, sendo que este decréscimo é mais pronunciado a partir de $30 \mathrm{phr}$ da carga mineral. Este comportamento corrobora os resultados mecânicos anteriores, que mostram 30phr como valor limite de carga na formulação em estudo.

Outros resultados mecânicos estão na Tabela 3. Uma característica da incorporação de teores crescentes de uma carga mineral na borracha natural é a redução das propriedades elásticas como alongamento, resiliência e deformação permanente à compressão (DPC), em comparação às propriedades da composição sem carga. Essa diminuição das propriedades

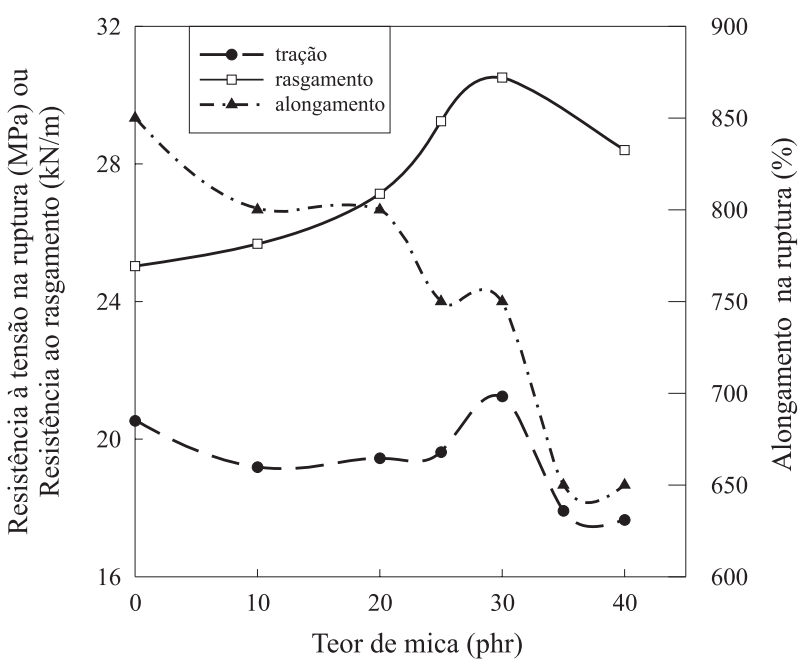

Figura 1. Resistência à tensão e ao rasgamento das composições NR/mica

elásticas é tanto maior quanto mais alto for o teor de mica incorporado à NR. Pode ser observado ainda na Tabela 3 que a adição crescente de mica aumenta a perda por abrasão das composições de borracha natural, o que é uma provável conseqüência do formato laminar da mica que facilita sua retirada da matriz borrachosa, pelo tipo de teste realizado (ASTM D 1044).

A Figura 2 apresenta os resultados de dureza, módulo a $300 \%$ e densidade de ligações cruzadas. Estes valores foram agrupados por estarem relacionados com a rigidez do compósito final. Comparando os resultados obtidos variando-se os teores de mica de 0 a $40 \mathrm{phr}$ observa-se que, quanto maior a quantidade de mica, mais elevados são os valores para as respectivas propriedades, com exceção para a formulação com 40phr indicando excesso desta carga mineral na composição.

A morfologia das diferentes composições está sendo estudada, na tentativa de se justificar os resultados obtidos, principalmente para teores superiores a 30phr. Estes resultados indicam uma mudança na morfologia das composições, causada não somente pela quantidade de mica, mas também pela dificuldade da distribuição e/ou dispersão desta carga, de natureza mineral, em uma matriz orgânica.

O cálculo da densidade de ligações cruzadas pode ser feito por vários métodos como: a partir de dados de tensão-deformação, de valores de módulo na região borrachosa, ou por inchamento em solventes orgânicos, sendo este último o adotado

Tabela 3. Propriedades mecânicas das composições NR/mica

\begin{tabular}{cccc}
\hline $\begin{array}{c}\text { Teor de mica } \\
(\mathbf{p h r})\end{array}$ & $\begin{array}{c}\text { Perda por abrasão } \\
(\mathbf{g} / \mathbf{1 0 0 0} \text { ciclos })\end{array}$ & $\begin{array}{c}\text { Resiliência } \\
(\boldsymbol{\%})\end{array}$ & $\begin{array}{c}\text { DPC } \\
(\boldsymbol{\%})\end{array}$ \\
\hline 0 & 0,16 & 65 & 16,7 \\
10 & 0,39 & 62 & 17,2 \\
20 & 0,48 & 64 & 17,3 \\
30 & 0,61 & 61 & 18,5 \\
40 & 0,82 & 59 & 22,6 \\
\hline
\end{tabular}




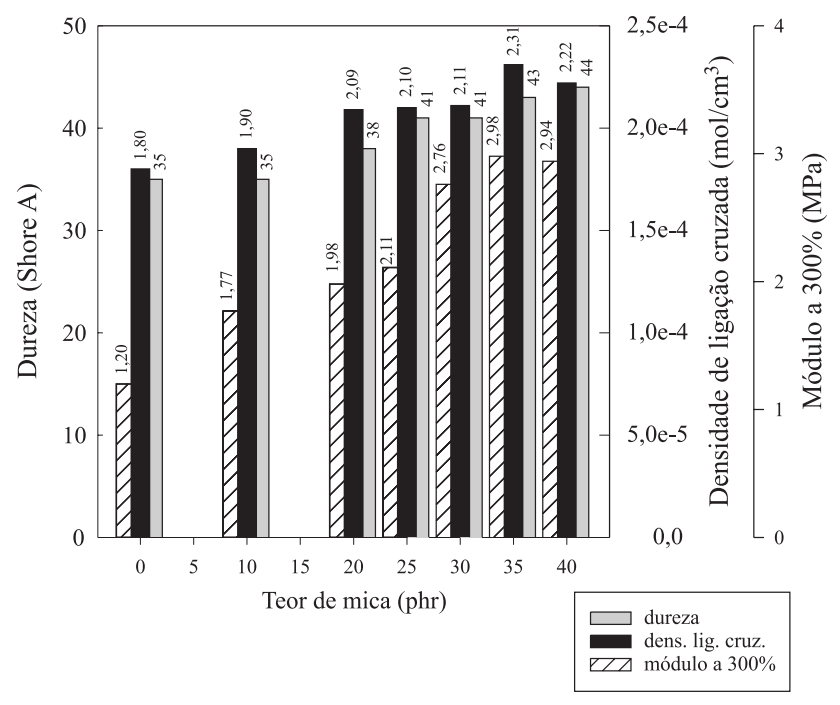

Figura 2. Dureza, módulo e densidade de ligações cruzadas das composições NR/mica

neste trabalho. Todos os diferentes métodos levam em consideração, tanto contribuições devido às ligações cruzadas químicas, quanto contribuições físicas ${ }^{[17]}$. Neste trabalho o que se observa são fracas contribuições físicas, inferindo no aumento pouco pronunciado no número de ligações cruzadas com o teor de mica na borracha natural, o que está de acordo com o desempenho mecânico, também pouco afetado, apresentado pelas composições $\mathrm{NR} /$ mica, quando comparados com a composição sem carga.

As curvas obtidas por DMTA para tan $\delta$ e módulo elástico das composições de borracha natural são mostradas nas Figuras 3 e 4, respectivamente. Na Figura 3 é possível observar que as composições $\mathrm{NR} /$ mica mostram uma ligeira tendência a menores valores de $T_{g}$, principalmente para as composições com 10 e $20 \mathrm{phr}$ de carga, em comparação à NR goma. Este comportamento parece indicar que o tipo de dispersão e teor da mica na matriz elastomérica podem estar relacionados com a maior facilidade desta carga, que é laminar, em afastar as cadeias macromoleculares, um comportamento semelhante ao de um plastificante. Vale ressaltar que a composição com $20 \mathrm{phr}$ de mica tem comportamento dinâmico melhor que aquela com $10 \mathrm{phr}$, o que pode ser verificado pela comparação das curvas de $\tan \delta$ de ambas as composições, e este resultado está de acordo com os resultados mecânicos quanto à resistência à tração destas duas composições. Na verdade, 10phr mostra ser um valor inferior ao necessário para que surjam contribuições mecânicas mais significativas. A composição com 30phr de mica apresentou menor tendência ao deslocamento da $\mathrm{T}_{\mathrm{g}}$. É interessante enfatizar que todas as composições com mica apresentaram melhor processamento, como já discutido. Logo os resultados de DMTA quanto ao posicionamento da $\mathrm{T}_{\mathrm{g}}$ estão inteiramente de acordo com este comportamento. Pode-se observar ainda que a adição crescente de mica provocou o decréscimo gradual da $\tan \delta$ das composições NR/ mica, em relação à NR goma. Este resultado é conseqüência do aumento do módulo elástico das composições de NR

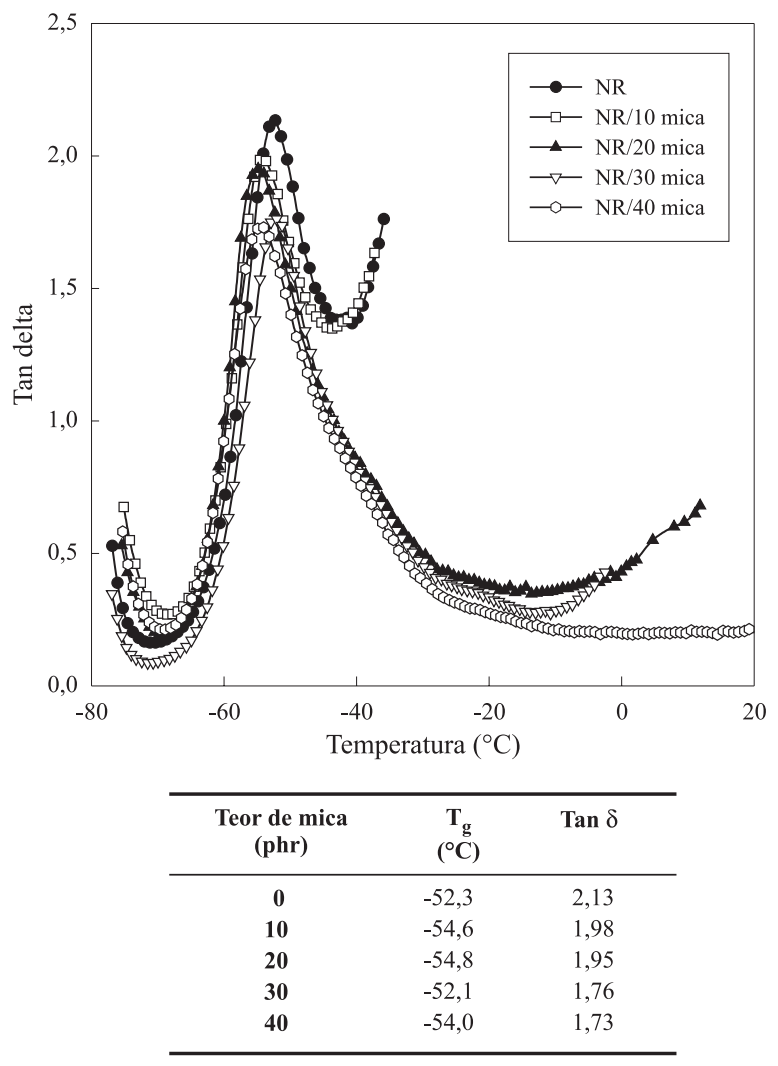

Figura 3. Tan $\delta$ versus temperatura de composições NR/mica

com a incorporação da carga, uma vez que a $\tan \delta$ é a relação entre os módulos viscoso (E") e elástico (E').

Da Figura 4 é possível observar que o efeito da incorporação de teores crescentes de mica à borracha natural é mais significativo na região borrachosa do que nas regiões vítrea e de transição, quanto ao módulo elástico. $\mathrm{O}$ comportamento das composições NR/mica segue a mesma tendência da densidade de ligações cruzadas. É possível também observar que a composição com $30 \mathrm{phr}$ tem maior módulo elástico na região borrachosa e começa a perder estabilidade em torno de $-10{ }^{\circ} \mathrm{C}$, o que acontece antes com a composição com $20 \mathrm{phr}$ de mica, e após com a composição com $40 \mathrm{phr}$ da carga mineral. Vale ressaltar que a estabilidade térmica da composição com 40phr de mica deve estar relacionada principalmente ao elevado teor de carga utilizado, o que não significa que esta composição apresente melhor desempenho mecânico limite (tensão na ruptura e resistência ao rasgamento).

\section{Conclusões}

- As propriedades físico-mecânicas sugerem que a interação da mica com a matriz de borracha natural não é de natureza química.

- O melhor desempenho quanto à resistência ao rasgamento e à tensão na ruptura foi alcançado pela composição de borracha natural com $30 \mathrm{phr}$ de mica, superior ao da NR.

- A presença de mica em NR facilita o processamento e aumenta a velocidade de vulcanização das misturas.

- As composições vulcanizadas NR/mica apresentam 


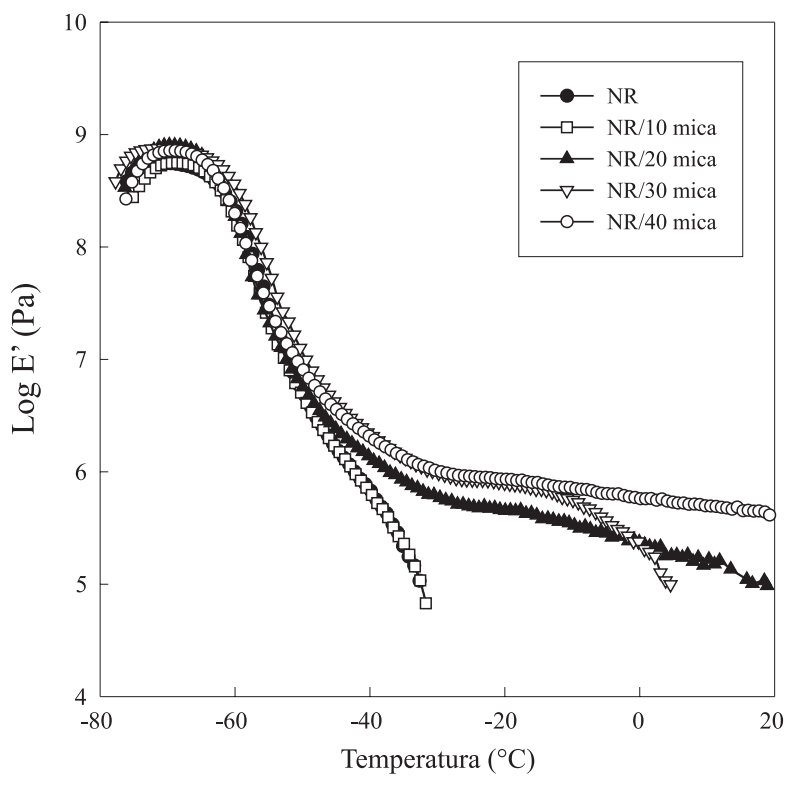

Figura 4. Módulo elástico versus temperatura de composições NR/mica

rigidez superior à NR, comprovada pelos ensaios de dureza, densidade de ligações cruzadas, resiliência e deformação permanente à compressão.

- Os testes dinâmico-mecânicos estão de acordo com as propriedades físico-mecânicas avaliadas.

\section{Agradecimentos}

Os autores agradecem ao Conselho Nacional de Desenvolvimento Científico e Tecnológico $(\mathrm{CNPq})$ e à Fundação Universitária José Bonifácio (FUJB) pelo apoio financeiro, e a Brasilminas Indústria e Comércio Ltda pela doação da mica.

\section{Referências Bibliográficas}

1. Bhowmick, A. K.; Hall, M. M. \& Benarey, H. A. - "Rubber Products Manufacturing Technology", Marcel Dekker Inc., New York (1994).

2. Mark, J. E.; Erman, B. \& Eirich F. R. - "Science and Technology of Rubber" - Academic Press, New York (1994).

3. Jones, K. P. - J. Nat. Rubb. Res., 12 (3), p.186 (1997).
4. Jorge, R. M. - "Mica em Composições de Borracha Natural", Dissertação de Mestrado, Universidade Federal do Rio de Janeiro, Brasil (2000).

5. Story, W. S. \& Washabaugh, F. J. - "Fillers", in: Enciclopedia of Polymer Science and Engineering, vol 7, H.F. Mark; N.M. Bikales; C.G. Overberger \& G. Menges (ed.), John Willey and Sons Inc., New York (1985).

6. Furtado, C. R. G. - "Substituição Parcial de Negro de Fumo e Sílica por Mica em Composições Elastomérica de $S B R$ ", Tese de Doutorado, Universidade Federal do Rio de Janeiro, Brasil (1996).

7. Rothon, R. N. - "Particulate-Filler Composites", Longman Group, Manchester (1996).

8. Hepburn, D. M.; Kemp, I. J. \& Shields, A. J. - Elet. Ins. Mag. 16 (5) p.19 (2000).

9. http://mineral.galleries.com

10. Batista, C. M. - http://www.dnpm.gov.br, 2000.

11. Wypych, G. - "Handbook of Fillers", Chem. Tech. Publ., New York, (1999).

12. Shell, H. R. - "Mica", in Enciclopedia of Chemical Technology, vol 13, Kirk Othmer (ed.), John Willey and Sons Inc., New York (1965).

13. Fekete, E.; Puranszky, B.; Toth, A. \& Bertoti,I. - J. Coll. Interf. Sci 135 (1) p.200 (1990).

14. Mathew, G.; Thomas, S. \& Kuriakose, B. - J. Elast. \& Plast., 29, p.163 (1997).

15. Ullman, R. - "Rubber Elasticity”, in: Structure and Properties of Polymers, cap. 8, Cahn, R.W.; Haasen, P.\& Kramer, E.J. (ed.), VCH Publishers Inc., New York (1993).

16. Flory, P.J. "Principles of Polymer Chemistry", Cornel University Press, New York (1953).

17. Hangen, R.; Salmén, L. \& Stenberg, B. - J. Polym. Sci., B34, p.1197 (1996).

Recebido: $21 / 10 / 02$

Aprovado: 31/03/03 